\title{
Mendelian disease caused by variants affecting recognition of Z-DNA and Z-RNA by the Za domain of the double-stranded RNA editing enzyme ADAR
}

\author{
Alan Herbert ${ }^{1}$ \\ Received: 29 January 2019 / Revised: 2 June 2019 / Accepted: 11 June 2019 / Published online: 18 July 2019 \\ (c) European Society of Human Genetics 2019
}

\begin{abstract}
Variants in the human double-stranded RNA editing enzyme ADAR produce three well-characterized rare Mendelian Diseases: Dyschromatosis Symmetrica Hereditaria (OMIM: 127400), Aicardi-Goutières syndrome (OMIM: 615010) and Bilateral Striatal Necrosis/Dystonia. ADAR encodes p150 and p110 protein isoforms. p150 incorporates the Z $\alpha$ domain that binds left-handed Z-DNA and Z-RNA with high affinity through contact of highly conserved residues with the DNA and RNA double helix. In certain individuals, frameshift variants on one parental chromosome in the second exon of ADAR produce haploinsufficiency of p150 while maintaining normal expression of p110. In other individuals, loss of p150 expression from one chromosome allows mapping of $Z \alpha$ p150 variants from the other parental chromosome directly to phenotype. The analysis reveals that loss of function $Z \alpha$ variants cause dysregulation of innate interferon responses to double-stranded RNA. This approach confirms a biological role for the left-handed conformation in human disease, further validating the power of Mendelian genetics to deliver unambiguous answers to difficult questions. The findings reveal that the human genome encodes genetic information using both shape and sequence.
\end{abstract}

\section{Introduction}

The ADAR genes produces three pre-mRNA transcripts, each initiated from a different promoter (Fig. 1 with exons numbered as in Kawakubo and Samuel, 2000 [1]). They code for the p150 and p110 protein isoforms [1]. The mRNA for p150 includes exon $1 \mathrm{~A}$ with its AUG translational start site $[2,3]$. p110 is encoded by mRNAs incorporating either exon $1 \mathrm{~B}$ or exon $1 \mathrm{C}$ spliced to exon 2 with its alternative AUG start site. Although only p150 has the $\mathrm{Z} \alpha$ domain, both $\mathrm{p} 150$ and $\mathrm{p} 110$ have a $\mathrm{Z} \beta$ domain, three double-stranded RNA binding domains and an enzymatic domain that catalyzes the deamination of adenosine to form inosine, which is subsequently readout as guanosine in RNA processing events (Fig. 1) [4].

Alan Herbert

alan.herbert@insideoutbio.com

1 Discovery, InsideOutBio, Inc 42 8th Street, Unit 3412, Charlestown, MA 02129, USA

\section{Materials and methods}

The strategy for mapping $Z \alpha$ variants to phenotypes focuses on affected individuals who are compound heterozygotes for the ADAR gene and who produce a haploid ADAR transcriptome (Fig. 1). The approach exploits variants on one allele that cause nonsense mediated decay of the RNA they produce. The variants on the other allele then map directly to phenotype as there is no transcript from the other chromosome to offset their effects.

\section{Results}

Variants that lead to p150 loss without affecting p110 expression are reported for two cases of Dyschromatosis Symmetrica Hereditaria (DSH), which is a disease of hypoand hyper-pigmentation of the dorsal surfaces of hands and feet with facial freckling. The disease is most common in Chinese and Asian populations. Inheritance characteristically follows an autosomal dominant mode of transmission, indicating that only one of the parental chromosomes carries the risk allele. Many of the disease variants introduce a frameshift stop codons (fs*) that leads 


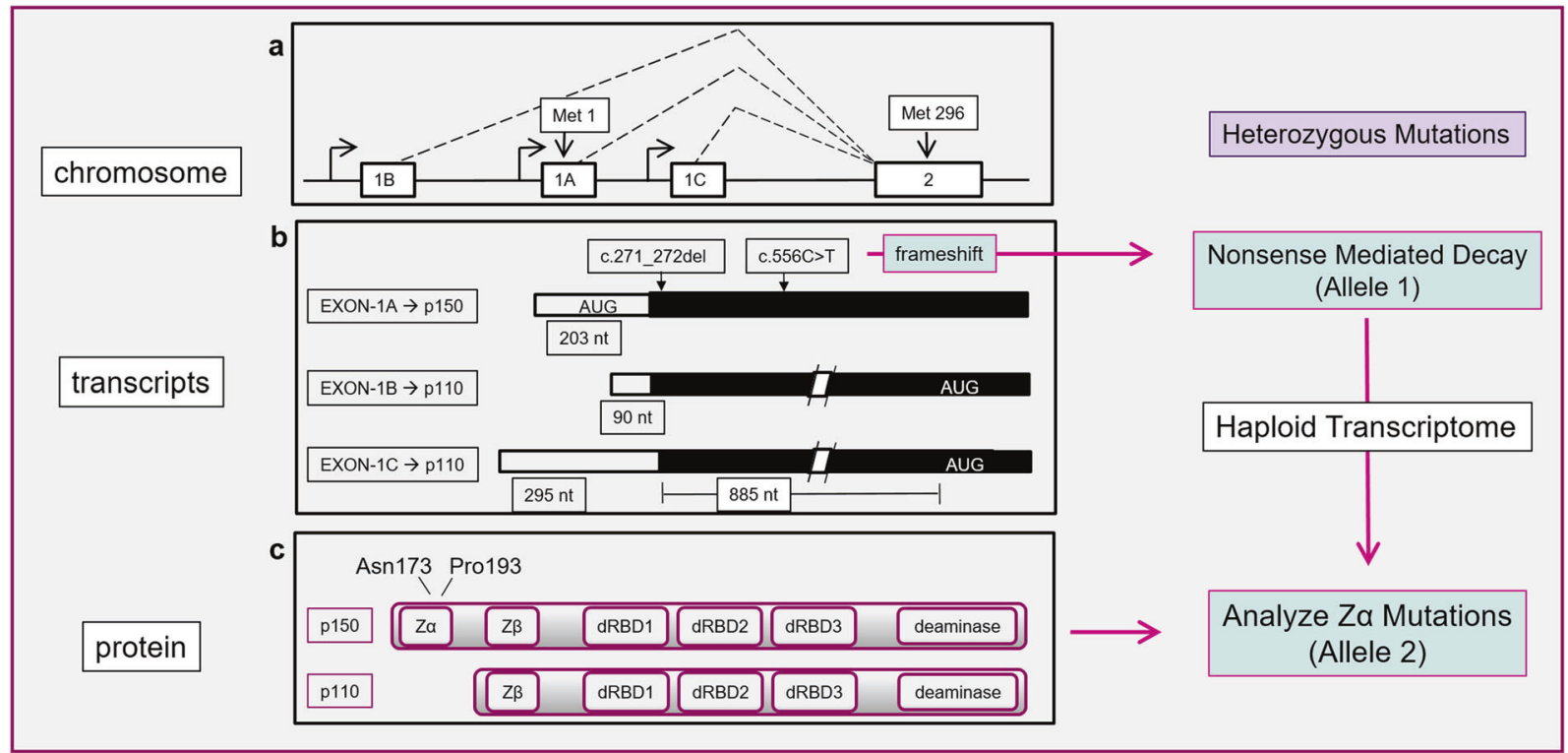

Fig. 1 a Exon structure of the Human ADAR gene with location of initiator methionine codons [1]. b Transcripts from the Human ADAR gene endcoding $\mathrm{p} 150$ and $\mathrm{p} 110$ protein isoforms. Variants c.271_272del and c.556c >t (reference sequence NM_001111.4, but with numbering starting at the translation initiation codon) introduce frameshifts leading to nonsense mediated decay of the mRNA produced from that allele, causing haploinsufficiency of p150. c Domain structure of $\mathrm{p} 150$ and $\mathrm{p} 110$ proteins. Variants $\mathrm{p} 150^{\mathrm{N} 173 \mathrm{~S}}$ and $\mathrm{p} 150^{\mathrm{P} 193 \mathrm{~A}}$

to nonsense mediated decay of the transcript [5]. In two well characterized, three generational pedigrees, frameshifts produce specific loss of p150 ("c.271_272del p. Arg91fs*123") [6] and ("c.556C > T p.Gln186*" [7]) (databases.lovd.nl/shared/variants/ADAR/unique, patient IDs 00230643 and 0000231740 ), with the RNA numbering from NM_001111.5, setting position 1 as the adenosine of the translation initiation codon, and amino acid numbering from www.uniprot.org, ID P55265-1), while leaving mRNA [6] and protein expression of $110^{7}$ unaffected. These cases indicate that allelic insufficiency of a p150 on a wildtype background is enough to cause this disease, even when p110 is expressed at normal levels. The dominant mode of transmission reflects this diminished expression of p150 in these individuals.

Aicardi-Goutières syndrome (AGS) and Bilateral Striatal Necrosis/Dystonia (BSD) are autosomal recessive diseases. AGS is classified as a type I Interferonopathy associated with increased expression of interferon-stimulated genes [8]. The phenotype is highly variable and overlaps that of BSD [9]. BSD is an early onset disease, characterized by developmental regression, dystonia, choreoathetosis, spastic quadriparesis, and cerebral calcification, often triggered by an infective or febrile episode [10]. In both diseases, frameshift alleles exist that lead to selective loss of p150 mRNA from one parental chromosome [8, 10] without affecting expression of wildtype p110 mRNA (haplotype lie in the $\mathrm{Z} \alpha$ domain. There are three double-stranded RNA binding domains (dRBD) and a catalytic domain (deaminase). The right-hand panel illustrates the general strategy used for mapping $\mathrm{Z} \alpha$ function in compound heterozyotes. The approach exploits variants on one allele that cause nonsense mediated decay of the RNA they produce. The variants on the other allele then map directly to phenotype as there is no normal p150 to offset their effects

$\left.\mathrm{p} 150^{\mathrm{fs} *} \mathrm{p} 110^{+}\right)[6,7]$. This background allows mapping of the phenotypic effects of $Z \alpha$ variants expressed from the other parental chromosome as there is no normal p150 present to offset them. In contrast, p110 variants are masked by wildtype $\mathrm{p} 110$ expressed from the $\mathrm{p} 150^{\mathrm{fs} *} \mathrm{p} 110^{+}$allele as is true for a disease where transmission is recessive.

Variants of the $\mathrm{Z} \alpha$ domain on a $\mathrm{p} 150^{\mathrm{fs} \mathrm{s}^{*}} \mathrm{p} 110^{+}$background are reported in one case of AGS [8] and in five cases of sporadic BSD [10]. The Z $\alpha$ variants occur at two different sites, Asn173 and Pro193 (numbering from NP_056655.2), both of which are essential to the interaction with Z-DNA and Z-RNA [11] (Fig. 2): Asn173 hydrogenbonds to the phosphate backbone while P193 forms hydrophobic contacts with the left-handed helix [12]. The variant NM_001111.4:c.577 C > A(p.(Pro193Ala)) (p150 ${ }^{\mathrm{P} 193 \mathrm{~A}}, \mathrm{dbSNP}$ ID rs145588689), which impairs editing by p150 [13], occurs in five patients [8], whereas NM_001111.4:c.518 A > G(p.(Asn173Ser)) (p150 ${ }^{\mathrm{N} 173 \mathrm{~S}}$, dbSNP ID rs201331183) is present in one individual [10]. In each case, p150 expression from one parental allele is lost, whereas the variant p150 transcript from the other parental allele produces a protein that no longer binds Z-DNA or Z-RNA. p110 expression from either parental chromosome is not affected. The AGS and BSD patient genotypes are either $\mathrm{p} 150^{\mathrm{P} 193 \mathrm{~A} / \mathrm{s}^{*}} \mathrm{p} 110^{+/+}$or $\mathrm{p} 150^{\mathrm{N} 173 \mathrm{~S} / \mathrm{s}^{*}} \mathrm{p} 110^{+/+}$in contrast with DSH individuals who have a $150^{+/ \mathrm{fs}^{*}} \mathrm{p} 110^{+/+}$genotype. Any phenotype owing 


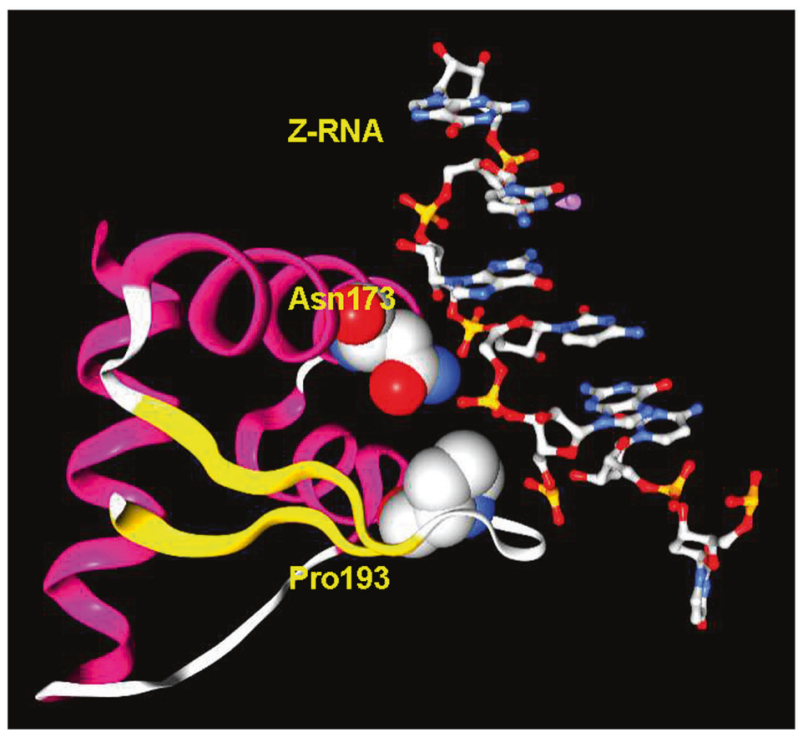

Fig. 2 The crystal structure of $\mathrm{Z} \alpha$ and Z-RNA. The direct contacts between p150 Asparagine(Asn)173 and p150 Proline(Pro)193 and the helix are highly conserved and essential for high affinity interaction (numbering from NP_056655.2 and structure from PDB: 2GXB [20])

to loss of Z-binding then becomes the difference between the six cases with AGS and BSD and the collection of individuals with DSH. It is apparent that the loss of Zbinding by variant $Z \alpha$ produces the dysregulation of interferon-stimulated genes found in AGS and BSD, but absent in DSH.

These findings from the human Mendelian catalog of diseases are consistent with mouse models where Adar deletion results in embryonic death attributable to excessive activation of innate interferon responses (reviewed in [14]). In mice with either homogeneous null $\left(\mathrm{Adar}^{-1-}\right)$ or with editing-deficient ADAR alleles (Adar ${ }^{E 861 A / E 861 A / E}$, numbering from www.uniprot.org, ID $=$ Q99MU3), the accumulation of endogenous double-stranded RNAs leads to activation of interferon-stimulated gene responses through a pathway dependent upon the melanoma differentiation associated gene 5 (MDA5, encoded by Ifhil). Inactivation of both Adar and Ifhil rescues the mouse phenotype, but Adar ${ }^{\text {p150-p } 150-}$ mutants that are unable to produce p150 do not (reviewed in ref. [14]).

The $\mathrm{p} 150^{\mathrm{P} 193 \mathrm{~A}}$ ADAR variant is present in $0.2 \%$ of individuals worldwide [15], and is highest in non-Finnish Europeans, approaching $0.3 \%$. The $\mathrm{p} 150^{\mathrm{N} 173 \mathrm{~S}}$ variant is highest in Finnish Europeans, also with a frequency of $0.3 \%$. ADAR is used by a number of viruses to enhance their virulence, including hepatitis delta virus, human immunodeficiency virus type 1 , vesicular stomatitis virus, and measles virus [16]. In locations where those infections are common, diminished ADAR function may favor individual survival by allowing a more robust immune response while diminishing viral replication [17]. Of note are the medieval measles epidemics that occurred once European cities became large enough to sustain outbreaks of disease. Selection by the measles virus may account for the higher frequency of $Z \alpha$ variants found in Europeans [18].

\section{Discussion}

The experiments of nature described here end the long drawn out dispute as to whether the Z-conformation has any biological relevance [14]. Clearly, variants in the $Z \alpha$ domain that diminish Z-DNA and Z-RNA binding causally affect the regulation of innate immune responses in man. There remain many open questions: why is the striatum most vulnerable to diminished p150 function in humans, why does allelic insufficiency of p150 impact neural crest derived melanoblast differentiation and is ADAR a therapeutic target for modulation of interferon responses? [19]

\section{Compliance with ethical standards}

Competing interests The author declares no competing interests.

Publisher's note: Springer Nature remains neutral with regard to jurisdictional claims in published maps and institutional affiliations.

\section{References}

1. Kawakubo K, Samuel CE. Human RNA-specific adenosine deaminase (ADAR1) gene specifies transcripts that initiate from a constitutively active alternative promoter. Gene. 2000;258: 165-72.

2. Kim U, Wang Y, Sanford T, Zeng Y, Nishikura K. Molecular cloning of cDNA for double-stranded RNA adenosine deaminase, a candidate enzyme for nuclear RNA editing. Proc Natl Acad Sci USA. 1994;91:11457-61.

3. Herbert A, Alfken J, Kim YG, Mian IS, Nishikura K, Rich A. A Z-DNA binding domain present in the human editing enzyme, double-stranded RNA adenosine deaminase. Proc Natl Acad Sci USA. 1997;94:8421-6.

4. Nishikura K. A-to-I editing of coding and non-coding RNAs by ADARs. Nat Rev Mol Cell Biol. 2016;17:83-96.

5. Li M, Yang L, Li C, Jin C, Lai M, Zhang G, et al. Mutational spectrum of the ADAR1 gene in dyschromatosis symmetrica hereditaria. Arch Dermatol Res. 2010;302:469-76.

6. Zhang JY, Chen XD, Zhang Z, Wang HL, Guo L, Liu Y, et al. The adenosine deaminase acting on RNA 1 p150 isoform is involved in the pathogenesis of dyschromatosis symmetrica hereditaria. Br J Dermatol. 2013;169:637-44.

7. Liu Q, Wang Z, Wu Y, Cao L, Tang Q, Xing X, et al. Five novel mutations in the ADAR1 gene associated with dyschromatosis symmetrica hereditaria. BMC Med Genet. 2014;15:69.

8. Rice GI, Kasher PR, Forte GM, Mannion NM, Greenwood SM, Szynkiewicz M, et al. Mutations in ADAR1 cause AicardiGoutieres syndrome associated with a type I interferon signature. Nat Genet. 2012;44:1243-8.

9. Kono M, Matsumoto F, Suzuki Y, Suganuma M, Saitsu H, Ito Y, et al. Dyschromatosis symmetrica hereditaria and Aicardi- 
Goutieres syndrome 6 are phenotypic variants caused by ADAR1 mutations. J Invest Dermatol. 2016;136:875-8.

10. Livingston JH, Lin JP, Dale RC, Gill D, Brogan P, Munnich A, et al. A type I interferon signature identifies bilateral striatal necrosis due to mutations in ADAR1. J Med Genet. 2014;51: 76-82.

11. Schade M, Turner CJ, Lowenhaupt K, Rich A, Herbert A. Structure-function analysis of the Z-DNA-binding domain Zalpha of dsRNA adenosine deaminase type I reveals similarity to the (alpha + beta) family of helix-turn-helix proteins. EMBO J. 1999;18:470-9.

12. Schwartz T, Rould MA, Lowenhaupt K, Herbert A, Rich A. Crystal structure of the Zalpha domain of the human editing enzyme ADAR1 bound to left-handed Z-DNA. Science. 1999; 284:1841-5.

13. Mannion NM, Greenwood SM, Young R, Cox S, Brindle J, Read $\mathrm{D}$, et al. The RNA-editing enzyme ADAR1 controls innate immune responses to RNA. Cell Rep. 2014;9:1482-94.

14. Herbert A. Z-DNAand Z-RNA in human disease. Commun Biol. 2019;2:7.
15. Lek M, Karczewski KJ, Minikel EV, Samocha KE, Banks E, Fennell T, et al. Analysis of protein-coding genetic variation in 60,706 humans. Nature. 2016;536:285-91.

16. Gelinas JF, Clerzius G, Shaw E, Gatignol A. Enhancement of replication of RNA viruses by ADAR1 via RNA editing and inhibition of RNA-activated protein kinase. J Virol. 2011;85: 8460-6.

17. Pfaller CK, Donohue RC, Nersisyan S, Brodsky L, Cattaneo R. Extensive editing of cellular and viral double-stranded RNA structures accounts for innate immunity suppression and the proviral activity of ADAR1p150. PLoS Biol. 2018;16:e2006577.

18. Black FL. Measles endemicity in insular populations: critical community size and its evolutionary implication. J Theor Biol. 1966;11:207-11.

19. Herbert A. ADAR and immune silencing in cancer. Trends Cancer. 2019;5:272-82.

20. Placido D, Brown BA 2nd, Lowenhaupt K, Rich A, Athanasiadis A. A left-handed RNA double helix bound by the $\mathrm{Z}$ alpha domain of the RNA-editing enzyme ADAR1. Structure. 2007;15: 395-404. 